\title{
A Numerical Study on Miscible Viscoelastic Fingering Instability
}

\author{
H. Shokri", M.H. Kayhani, M. Norouzi \\ Mechanical Engineering Department, Shahrood University of Technology, Iran
}

Copyright $\bigcirc 2017$ by authors, all rights reserved. Authors agree that this article remains permanently open access under the terms of the Creative Commons Attribution License 4.0 International License

\begin{abstract}
In this study, the viscoelastic fingering instability of miscible displacement is investigated using computational fluid dynamics (CFD). The Maxwell's model has been used as the constitutive equation of viscoelastic fluid and Hartley transformation (as a spectral method) and fourth-order Adams-Bashforth technique are used to solve the governing equations. The development of the finger structures is discussed using concentration contours and diagrams of transversely average concentration, mixing length and sweep efficiency. It is found that the flow is more stabilized when relaxation time of displacing fluid is increased.
\end{abstract}

Keywords Fingering Instability, Viscoelastic Fluid, Spectral Method

\section{Introduction}

Viscous fingering has been known as one of the most important phenomena in displacement processes for decades. This refers to kind of instability that occurs in the displacement of fluids in porous materials. In most but not all cases, the mechanism of this instability is linked to the viscosity variation between two displacing and displaced fluids. By injecting a low viscous fluid into the porous medium that saturated with high viscous fluid, over time, the interface of two fluids will become unstable and the injected fluid will penetrate with finger-like patterns into the high viscous fluid. This instability has attracted much attention due to its role in a wide variety of natural systems and industrial processes such as hydrology, filtration and most importantly, processes of secondary and tertiary oil recovery.

The scientific study of this fingering instability, first was reported by Hill and Inst. P [1]in 1952. Then, the studies continued by many researchers in this field. Details of these studies up to 1987 are presented by Homsy[2]. In 1988, for the first time, a Fourier spectral method was used to study the nonlinear behavior of viscous fingering by Tan and Homsy[3]. Their results show a good agreement with their previous linear stability analysis[4]. Prior to this study, simulations of nonlinear viscous fingering were limited to use of low accuracy method such as finite difference method. In the flowing, Zimmerman and Homsy[5] performed Hartley spectral method to the simulation of viscous fingering. They examined the effect of anisotropic dispersion in miscible displacements. They observed new mechanisms such as multiple coalescence and fading in simulations with anisotropic dispersion, but not for isotropic dispersion. In the following, these authors[6] simulated multidimensional viscous fingering by an extension of the Hartley spectral methods to three dimensions. Their results show that two-dimensional simulations are sufficient to capture essential features of nonlinear viscous fingering.

The seminal experimental works on viscous fingering in the presence of non -Newtonian fluid in the flow were conducted by Nittman et al. [7] and Daccord et al. [8]. Then, study the effect of properties non-Newtonian fluid on fingering instability was followed by many researcher. The vast majority of these studies have focused on the role of shear-thinning fluid in this instability [9-11]. In 1964, Pye [12] and Sandiford [13] experimentally concluded that water mobility ratio could effectively be reduced by adding small amount of water soluble polymers. Typically, in the case of polymer solutions in Newtonian fluid (except in very dilute solutions), viscoelastic fluids can be obtained. Viscoelastic fluid behavior is between the fluid and solid. It is the viscoelastic fingering instability that this paper will focus.

In this paper, the effect of viscoelastic behavior on the viscous fingering is investigated. For this purpose, CFD simulation is used and concentration contours, Sweep efficiency, transversely average concentration, mixing length will be examined. Here, Maxwell's model is used as the constitutive equation of viscoelastic fluid.

\section{Formulation}

The schematic shape of problem is shown in Figure 1. 
According to the figure, A rectangular box with length of $L$ and width of $W$ is considered as the geometry of the problem.

Here, the low viscosity fluid $\left(\eta_{1}\right)$ is pushed in direction of box into the porous medium which is saturated with high viscous fluid $\left(\eta_{2}\right)$. The flow is considered miscible. In this study, the Darcy's law is considered for porous medium and it is possible to show that this supposition is identical with flow through the gap of two parallel plates (known as the horizontal rectilinear Hele-Shaw cell).

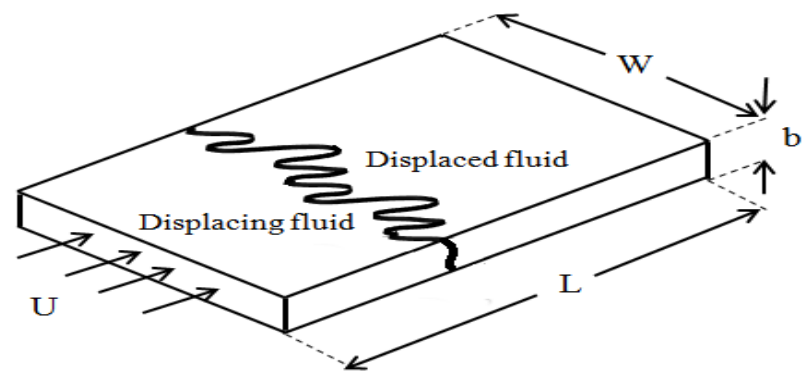

Figure 1. Schematic of flow system

The two-dimensional miscible displacement with incompressible Newtonian fluids is described by continuity equation, Darcy's law (as a momentum equation) and the convection-dispersion equation as following:

$$
\begin{gathered}
\nabla \cdot \mathbf{u}=0 \\
\nabla p=-\eta K^{-1} \mathbf{u} \\
\frac{\partial c}{\partial t}+\mathbf{u} \cdot \nabla c=\nabla \cdot(D \nabla c)
\end{gathered}
$$

Where $\mathbf{u}=(\mathrm{u}, \mathrm{v})$ is velocity vector. The other variables are the pressure $p$, the viscosity $\eta$, the permeability $K$, the dispersion coefficient $D$ and the concentration is displayed with $c$.

When the displacement of viscoelastic fluid is considered, the equation (2) needs to be modified. For this purpose, the Maxwell's model is considered as the constitutive equations of viscoelastic fluids. This model is implemented as follows:

$$
\begin{gathered}
\tilde{\tau}+\lambda \tilde{\tau}_{(1)}=\eta \dot{\gamma}_{(1)} \\
u^{*}\left(x^{*}=-t^{*}, y^{*}, t^{*}\right)=1, \quad v^{*}\left(x^{*}=-t^{*}, y^{*}, t^{*}\right)=0, \quad c^{*}\left(x=-t^{*}, y^{*}, t^{*}\right)=1 \\
u^{*}\left(x^{*}=P e-t^{*}, y^{*}, t^{*}\right)=1, \quad v^{*}\left(x^{*}=P e-t^{*}, y^{*}, t^{*}\right)=0, \quad c^{*}\left(x^{*}=P e-t^{*}, y^{*}, t^{*}\right)=0 \\
\left(u^{*}, c^{*}\right)\left(x^{*}, y^{*}=0, t^{*}\right)=\left(u^{*}, c^{*}\right)\left(x^{*}, y^{*}=A, t^{*}\right)
\end{gathered}
$$

Where $\tilde{\tau}$ and $\tilde{\tau}_{(1)}$ are the stress tensor and upper convected derivative of the stress tensor, respectively. By analogy of with Maxwell's model, the modified Darcy's law [14]has been introduced:

$$
\left(1+\lambda \frac{\partial}{\partial t}\right) \nabla p=-\frac{\eta}{K} \mathbf{u}
$$

Here, the boundary and initial conditions are defined as follows:

$$
\begin{gathered}
u(x=L, y, t)=U \quad v(x=L, y, t)=0 \quad c(x=L, y, t)=0 \\
(u, c)(x, y=0, t)=(u, c)(x, y=W, t) \\
u\left(x, y, t=t_{0}\right)=U, v\left(x, y, t=t_{0}\right)=0, \quad c\left(x, y, t=t_{0}\right)=c_{0}
\end{gathered}
$$

In order to make non-dimensional governing equations, $D / U$ and $D / U^{2}$ are used as reference length and time, respectively.

In addition, the viscosities of both displacing and displaced fluids and also the velocity, concentration and pressure are scaled by $\eta_{1}, U, c_{1}$ and $\eta_{1} U^{2} / D$, respectively. Also, a Lagrangian reference frame that moving with superficial velocity $U$ is chosen to study of the flow.

Therefore, the dimensionless forms of governed equations can be expressed as:

$$
\begin{gathered}
\nabla \cdot \mathbf{u}^{*}=0 \\
\left(1+\lambda^{*} \frac{\partial}{\partial t^{*}}\right) \nabla p^{*}=-\frac{\eta^{*}}{K^{*}}\left(\mathbf{u}^{*}+i\right) \\
\frac{\partial c^{*}}{\partial t^{*}}+\mathbf{u}^{*} \cdot \nabla c^{*}=\nabla^{2} c^{*}
\end{gathered}
$$

In non-dimensional modified Darcy's law equation, “ $i$ ” is the unit vector in the $x$-direction that has arisen due to Lagrangian reference frame and $\lambda^{*}$ defined as $\lambda_{1} U^{2} / D$. 
In these equations, $P e=L U / D$ is the Peclet number for mass transfer, this parameter represents the ratio of the convective forces over diffusive forces and $A=L / W$ is the aspect ratio.

For convenience, in following, the star superscript that used to show non-dimensional parameters is omitted.

To complete the model, as same as Zimmerman and Homsy[5], an exponential relationship between the viscosity and the concentration is selected:

$$
\eta(c)=e^{R(1-c)}
$$

Where

$$
R=\ln \left(\eta_{2} / \eta_{1}\right)
$$

To complete the model, the similar exponential relations is proposed for $\lambda(c)$.

$$
\lambda(c)=\lambda_{1} e^{R^{\prime}(1-c)}
$$

Where

$$
R^{\prime}=\ln \left(\lambda_{2} / \lambda_{1}\right)
$$

In the above equation, $\lambda_{2}$ and $\lambda_{1}$ are non-dimensional relation times of displaced and displacing fluids, respectively.

Following the earlier studies $[3,15,16]$, the well-known stream function-vorticity formulation is used to solve the equations describing the flow as:

$$
\begin{gathered}
u=\frac{\partial \psi}{\partial y}, \quad v=-\frac{\partial \psi}{\partial x} \\
\omega=\frac{\partial v}{\partial x}-\frac{\partial u}{\partial y}=-\left(\frac{\partial^{2} \psi}{\partial x^{2}}+\frac{\partial^{2} \psi}{\partial y^{2}}\right)
\end{gathered}
$$

Where $\psi$ is the stream function and $\omega$ is the vorticity. Using this transformation, the continuity equation is automatically satisfied and the convection-dispersion equation can be obtained as follows:

$$
\frac{\partial c}{\partial t}+\frac{\partial \psi}{\partial y} \frac{\partial c}{\partial x}-\frac{\partial \psi}{\partial x} \frac{\partial c}{\partial y}=\frac{\partial^{2} c}{\partial x^{2}}+\frac{\partial^{2} c}{\partial y^{2}}
$$

Taking the curl of Eq. (10), we have:

$$
\omega=-\frac{R^{\prime}}{\frac{\eta}{K}} \frac{\partial c}{\partial y} \lambda \frac{\partial^{2} p}{\partial x \partial t}+\frac{R^{\prime}}{\frac{\eta}{K}} \frac{\partial c}{\partial x} \lambda \frac{\partial^{2} p}{\partial y \partial t}-R\left(\left(1+\frac{\partial \psi}{\partial y}\right) \frac{\partial c}{\partial y}-\frac{\partial c}{\partial x} \frac{\partial \psi}{\partial x}\right)
$$

In order to simulate the nonlinear viscous fingering instability, a very powerful tools is used for the numerical calculation which is known as the spectral method that has been widely used in fluid mechanics. Main advantage of spectral method is its high accuracy, which called spectral accuracy. In this method, by implementing modified Hartley transforms, the partial differential equation that describes the flow displacement recasts into an ordinary differential equation. The two dimensional discrete Hartley transform for an arbitrary function of $x$ and $y$ can be presented as:

$$
G\left(k_{x}, k_{y}\right)=\frac{1}{\sqrt{N_{x} N_{y}}} \sum_{i=1}^{N_{x}} \sum_{j=1}^{N_{y}} g(x, y) \operatorname{cas}\left(\frac{2 \pi x k_{x}}{N_{x}}+\frac{2 \pi x k_{y}}{N_{y}}\right)
$$

Where, $N_{x}$ and $N_{y}$ represent the number of grid points in $\mathrm{x}$ and $\mathrm{y}$ directions, $K_{x}$ and $K_{y}$ are the corresponding wavenumbers and cas $(u)=\sin (u)+\cos (u)$. Using spectral method and Hartly transform requires periodic boundary conditions but our concentration boundary conditions aren't periodic. To solve this problem, Manickam et al. [17] suggested a very efficient solution to split concentration at any time in space into two component; a base state profile $\bar{c}(x, t)$ and disturbance concentration, $c^{\prime}(x, y, t)$ that decay for boundaries of problem as follows:

$$
c(x, y, t)=\bar{c}(x, t)+c^{\prime}(x, y, t)
$$

Where $c^{\prime}(x, y, t)$ is:

$$
c^{\prime}(x, y, t)=\frac{1}{2}\left(1-\operatorname{erf}\left(\frac{x}{2 \sqrt{t}}\right)\right)
$$

Therefore, instead of solving the total concentration, only the disturbance concentration will be solved that have periodic boundary conditions. Also, initial condition for concentration is defined as:

$$
c_{0}\left(x, y, t=t_{0}\right)=\frac{1-\operatorname{erf}\left(\frac{x}{\sqrt{4 t}}\right)}{2}+\delta \text {.rand }(y) \cdot \exp \left(-\frac{x^{2}}{\sigma^{2}}\right)
$$

In this equation, rand is a random number between -1 and $1, \delta$ is the magnitude of the disturbance, $\sigma$ can be construed as the penetration of the disturbance from the front, $t_{0}$ is the initial time in which the perturbation concentration is added to the base state profile. Time marching in the concentration is provided by a fourth-order Adams-Bashforth predictor-corrector method. Using this technique, a semi-implicit algorithm and a stable time stepping are obtained. The time advancement in the concentration, vorticity and stream function are evaluated, respectively. The new obtained updated value is then used to find a new value to evaluate concentration field in the following time steps.

\section{Results}

In order to compare the results for viscoelastic flow, several useful quantities are introduced to characterize the properties of fingering phenomenon. Unless otherwise 
mentioned, the flows are analyzed for $P e=1000, A=2$, $R=3$.

\section{Transversely Averaged Profiles}

Transversely averaged profiles of concentration are a classical measurement in experimental and theoretical studies. Figures 2 and 3 show the variation of the transversely averaged concentration profiles as a function of time for $\lambda_{1}=1.5, \lambda_{2}=0.5$ in two different mobility ratio $\mathrm{R}$. it is clear that in initial time, since the fingers still are not very developed, the transversely profile continue to track the base state profile but when the fingers are grown up, some peaks were created in the profiles. Comparing two figures, It can be seen that the intensity of distortion is reduced by decreasing the mobility ratio. In fact, the viscosity difference between displacing and displaced fluids is known to main factor of fingering instability, therefore, it is natural that flow become more stable when mobility ratio decreases.

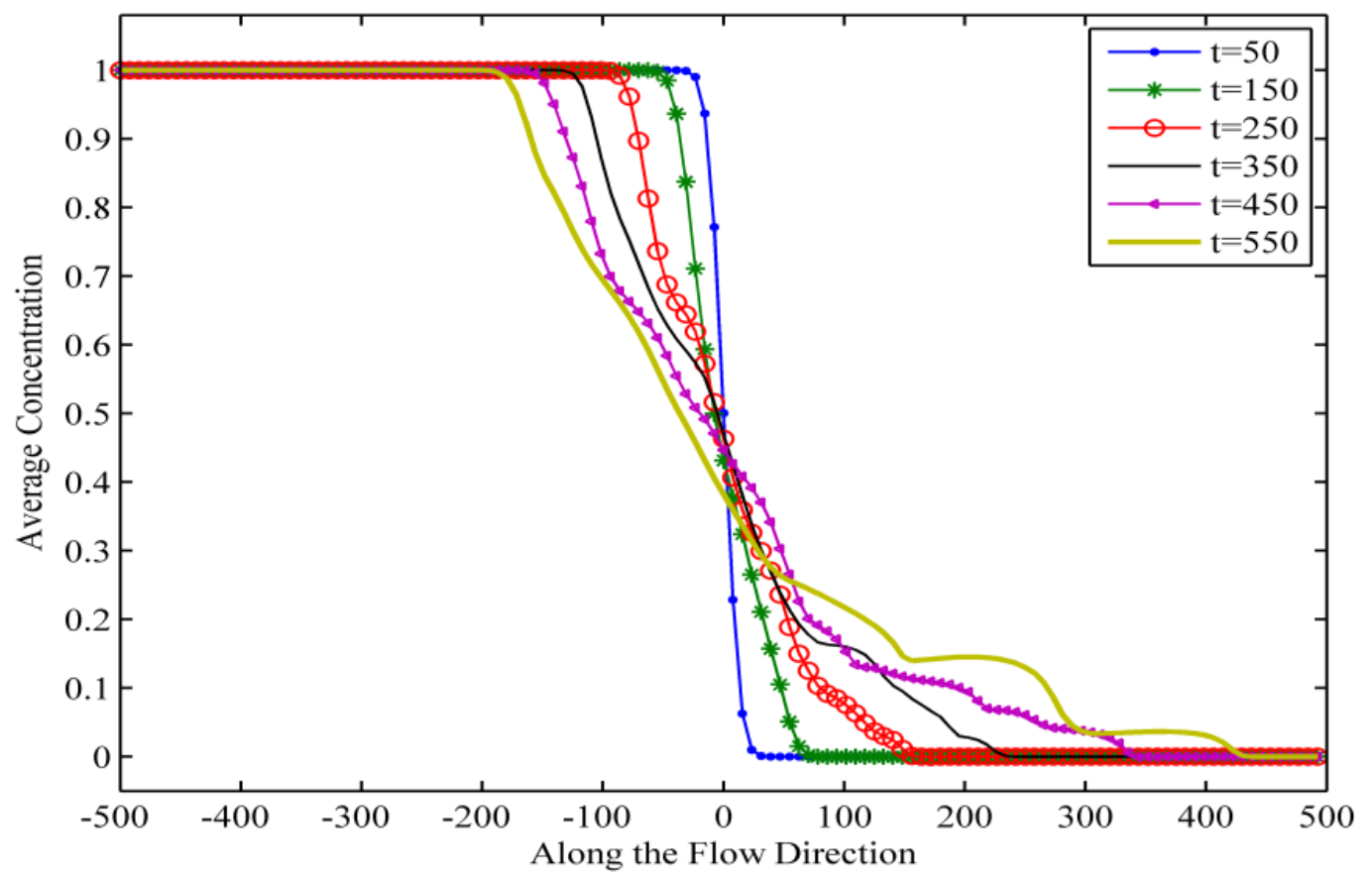

Figure 2. Transversely averaged concentration profiles at $\lambda_{1}=1.5, \lambda_{2}=0.5$ and $\mathrm{R}=3$.

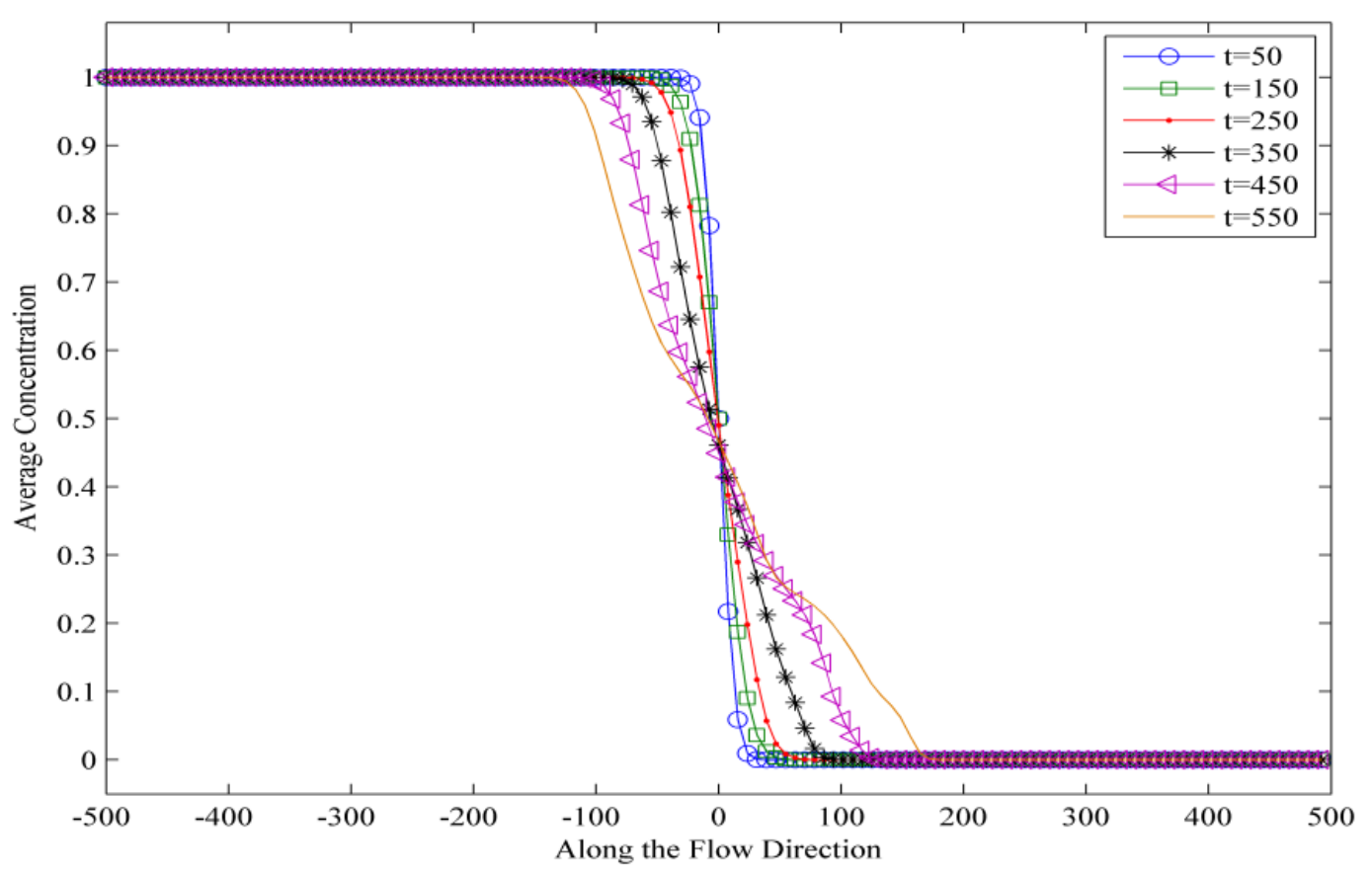

Figure 3. Transversely averaged concentration profiles at $\lambda_{1}=1.5, \lambda_{2}=0.5$ and $\mathrm{R}=2$. 


\section{Mixing Length}

The length of the fingering zone which is classically referred to as the "mixing length" in the viscous fingering studies is an important measurement of the region where the two fluids are mixed. there are several different ways to compute of it. In this study, the proposed method by Zimmerman and Homsy[5] is used. The mixing length is defined as the distance between $\bar{c}=1-\delta$ and $\bar{c}=\delta$ in the transversely averaged concentration profile:

$$
L_{\delta}=\left.x\right|_{\bar{c}=\delta}-\left.x\right|_{\bar{c}=1-\delta}
$$

In the above equation, $\delta$ is a small positive number and $\bar{c}$ is the transversely averaged concentration. Figure 4 shows the variation of the mixing length as a function of time and mobility ratio. As expected, the mixing length increases by increasing mobility ratio. Also, it is clear as the flow evolves, the mixing length increases monotonically with time. Figure 5 depicts the mixing length for $\lambda_{2}=0.5$ at different relaxation time of displacing fluid $\left(\lambda_{1}\right)$. Unlike the mobility ratio, the mixing length is decreased by increasing $\lambda_{1}$. Therefore, it can be said that the elasticity of viscoelastic fluid can be a stabilizing factor in this type of flow.

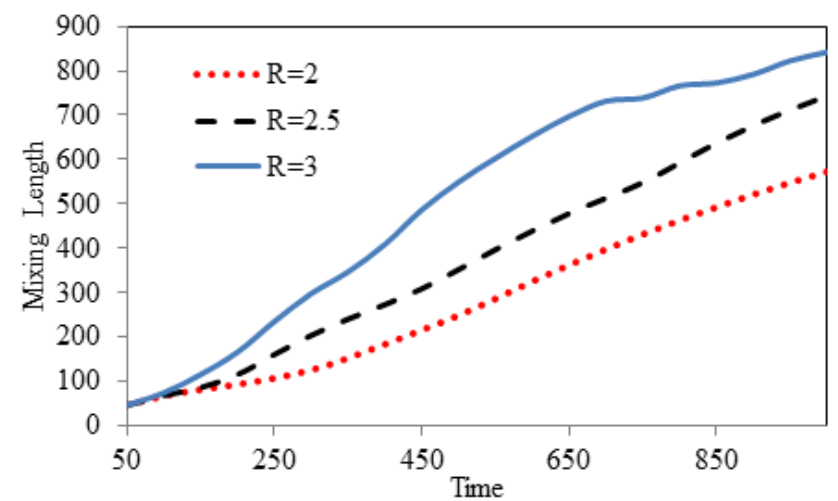

Figure 4. Mixing length profiles at $\lambda_{1}=1.5$ and $\lambda_{2}=0.5$.

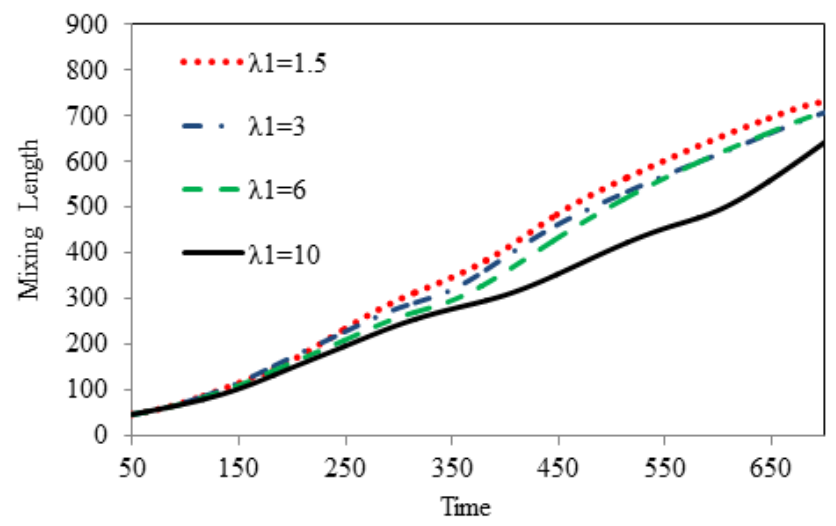

Figure 5. Mixing length profiles at $\lambda_{2}=0.5$ and $\mathrm{R}=3$.

\section{Sweep Efficiency}

One of the property that used widely in industry is sweep efficiency. This parameter shows how much the displacing flow has been successful in moving the displaced fluid. In this study, following the Ghesmat and Azaiez[15], it is defined as dividing the number of grid points where the concentration is equal to or larger than 0.5 by the total number of grid points that are located behind the front defined by $c=0.5$ in the fixed frame.

Figure 6 depicts sweep efficiency as function of mobility $R$ ratio and time. It can be seen that the sweep efficiency is increased by decreasing the mobility ratio. Also, Figure 7 shows the variation of sweep efficiency as function of $\lambda_{1}$. It is observed from the results that by advancement and increasing the number of fingers, the sweep efficiency decreases with time. Also, It is worth noting that the $\lambda_{1}$ is effective to increase the sweep efficiency.

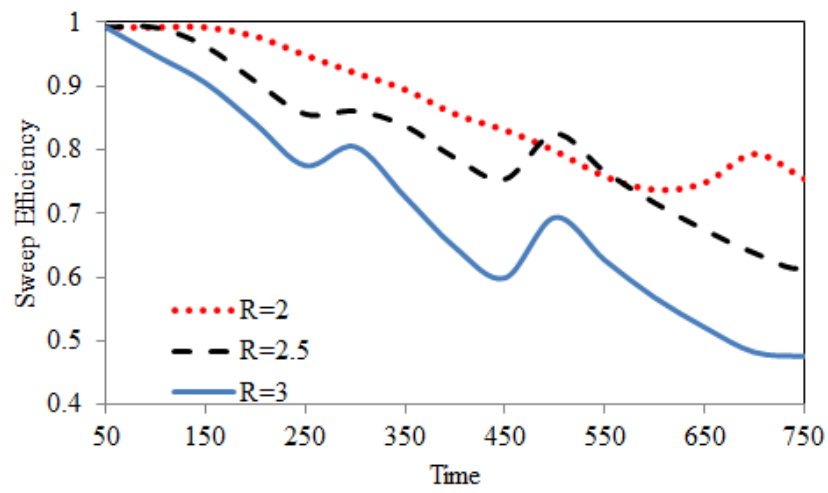

Figure 6. Sweep efficiency profiles at $\lambda_{1}=1.5$ and $\lambda_{2}=0.5$.

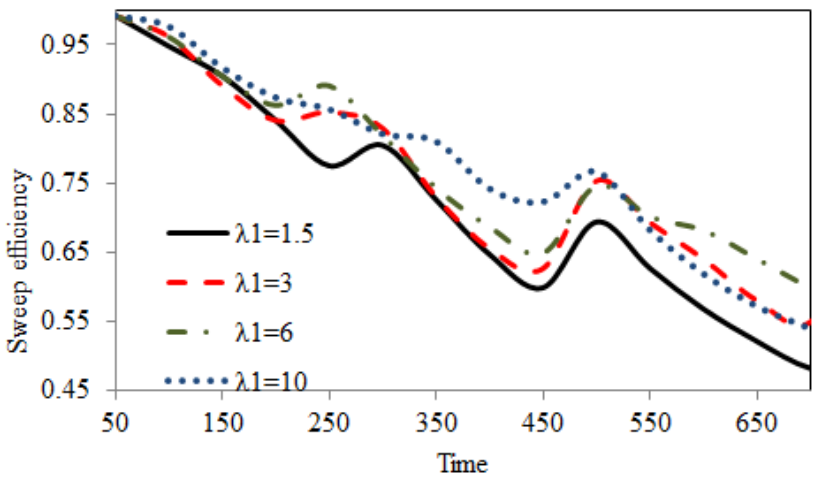

Figure 7. Sweep efficiency profiles at $\lambda_{2}=0.5$ and $\mathrm{R}=3$.

\section{Concentration Contours}

Figure 8 shows contours of the flow displacements for $\lambda_{1}=1.5$ and $\lambda_{2}=0.5$. In fact, the instability is caused by including random disturbances of small magnitude that are localized around the interface at the start of the simulations. Then, these disturbances quickly develop in to fingers that grow through different mechanisms. One of this mechanism that can be identified in these figures by a circle is called coalescence (the frames at $\mathrm{t}=500$ ).

In this mechanism, the tip of a finger is bent to the adjacent finger and finally merges into its body and a wider and longer finger is caused. 


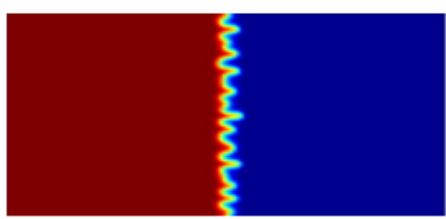

$\mathrm{t}=100$

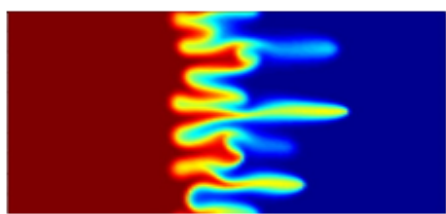

$\mathrm{t}=400$

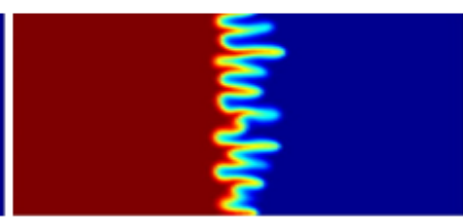

$\mathrm{t}=200$

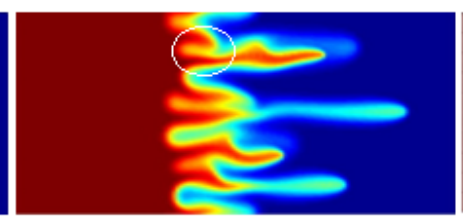

$\mathrm{t}=500$

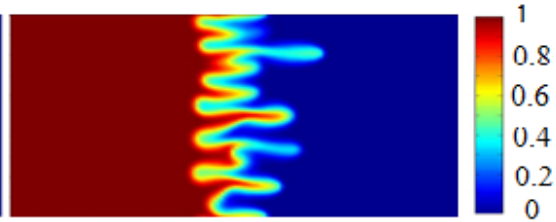

$\mathrm{t}=300$

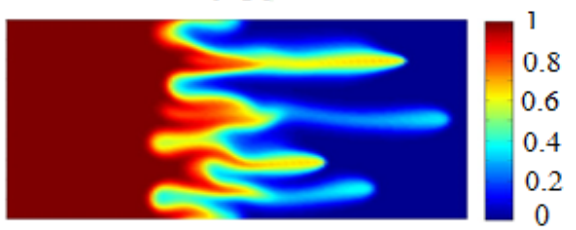

$\mathrm{t}=600$

Figure 8. concentration iso-contours at $\lambda_{1}=1.5$ and $\lambda_{2}=0.5$.

\section{Conclusions}

In this study, the nonlinear simulations is used to investigate the effects of viscoelastic fluid properties on fingering instability. Also, the flow was considered two-dimensional, incompressible and miscible and Maxwell's model is used as the constitutive equation of viscoelastic fluid. . The results conclude the concentration contours, transversely averaged profiles, mixing length profiles and sweep efficiency.it is concluded that the flow is more stable by increasing relaxation time $\left(\lambda_{1}\right)$ of displacing fluid.

\section{REFERENCES}

[1] Hill, S. (1952). Channeling in packed columns. Chemical Engineering Science, 1(6), 247-253.

[2] Homsy, G. M.(1987). Viscous fingering in porous media, Annual review of fluid mechanics, 19( 1), 271-311.

[3] Tan, C., \& Homsy, G. (1988) Simulation of nonlinear viscous fingering in miscible displacement, Physics of Fluids, 31( 6), 1330-1338.

[4] Tan, C., \& Homsy, G. (1986). Stability of miscible displacements in porous media: rectilinear flow, Physics of Fluids, 29( 11), 3549-3556.

[5] Zimmerman, W., \& Homsy, G. (1991), Nonlinear viscous fingering in miscible displacement with anisotropic dispersion, Physics of Fluids A: Fluid Dynamics, 3(8), 1859-1872.

[6] Zimmerman, W., \& Homsy, G. (1992), Three - dimensional viscous fingering: A numerical study, Physics of Fluids A: Fluid Dynamics, 4(9), 1901-1914.

[7] Nittmann, J., Daccord, G., \& Stanley, H. E. (1985), Fractal growth of viscous fingers: quantitative characterization of a fluid instability phenomenon, Nature, 314(6007), 141-144.
[8] Daccord, G., Nittmann, H., \& Stanley, H. E.(1986), Radial viscous fingers and diffusion-limited aggregation: Fractal dimension and growth sites, Physical review letters, 56(4), 336.

[9] Li, H., Maini, B., \& Azaiez, J. (2006), Experimental and Numerical Analysis of the Viscous Fingering Instability of Shear - Thinning Fluids, The Canadian Journal of Chemical Engineering, 84(1), 52-62

[10] Kim, M. C., \& Choi, C. K. (2011), Linear analysis on the stability of miscible dispersion of shear-thinning fluids in porous media, Journal of Non-Newtonian Fluid Mechanics, 166(21), 1211-1220.

[11] Shoghi, M. R., \& Norouzi (2015), M, Linear stability analysis and nonlinear simulation of non-Newtonian viscous fingering instability in heterogeneous porous media, Rheologica Acta, 54(11), 973-991.

[12] Pye, D. J. (1964), Improved secondary recovery by control of water mobility, Journal of Petroleum technology, 16(8), 911-916.

[13] Sandiford, B. (1964), Laboratory and field studies of water floods using polymer solutions to increase oil recoveries, Journal of Petroleum Technology, 16(8), 917-922.

[14] Khuzhayorov, B., Auriault, J. L., \& Royer, P. (2000), Derivation of macroscopic filtration law for transient linear viscoelastic fluid flow in porous media, International Journal of Engineering Science, 38(5), 487-504.

[15] Ghesmat, K., \& Azaiez, J. (2008), Viscous fingering instability in porous media: effect of anisotropic velocity-dependent dispersion tensor, Transport in Porous Media, 73(3), 297-318.

[16] Nourozi. M., \& Shoghi, M (2015), Nonlinear simulation of non-Newtonian viscous fingering instability in anisotropic porous media, Modares Mechanical Engineering, 15(7), 11. In Persian

[17] O. Manickam, O., \& G. Homsy, G. (1993), Stability of miscible displacements in porous media with nonmonotonic viscosity profiles, Physics of Fluids A: Fluid Dynamics, 5( 6), 1356-1367. 